\title{
UNKNOTTING IN $M^{2} \times I$
}

\author{
BY \\ E. M. BROWN
}

1. Introduction. Let $N$ be a manifold, $P \subset N$ a "naturally" embedded submanifold of $N$. An embedding $f: P \rightarrow N$ unknots in $N$ if there is a homeomorphism $h$ of $N$ onto itself such that

$$
h f(P)=P \subset N
$$

i.e. we can put $f(P)$ into the "natural" position of $P$ in $N$ via a homeomorphism of $N$. Classically $N$ is the 3-sphere and $P$ is a great circle in $N$. This case was solved by C. D. Papakyriakopoulos [6] in the sense that he reduced it to a computation of the fundamental group $\pi_{1}\left(S^{3}-f\left(S^{1}\right)\right)$.

We shall consider the case where $N=M^{2} \times I, M^{2}$ a compact 2-manifold and $I=[0,1]$. In this case there are several "natural" submanifolds of $N$ viz.;

(i) For $p_{1}, \cdots, p_{n}$ points in the interior of $M$, there is the natural submanifold $\bigcup_{j=1} p_{j} \times I$.

(ii) For $\alpha$ a simple arc in $M$, joining boundary points of $M$, the disk $\alpha \times I$.

(iii) For $\lambda$ a simple loop in the interior of $M$, the annulus $\lambda \times I$.

(iv) $M \times 1 / 2$.

We give necessary and sufficient conditions for unknotting in the above four cases. The condition we give for (i) is of the same nature as that of Papakyriakopoulos, and the theorem we prove is equivalent to his Theorem (28.1 i) of [6] when $n=1$ and $M$ is a disk. It was shown by Zeeman [11] that an arc regularly (see $\$ 2$ for definitions) embedded in a ball of dimension greater than three is always unknotted (this is a combinatorial theorem). One may well ask what the situation of (i) is when the dimension of $M$ is greater than two.

Case (iv) is also interesting in higher dimensions, in particular when $M$ is a sphere. A locally flat version of our (7.2) would establish the annulus conjecture. A solution of case (iv) identical to ours was obtained in [2] when $M$ is a closed 2-manifold.

In $\$ 2$ occur definitions and some elementary propositions which will be needed throughout the paper.

In $\S 3$ we shall prove the main geometric theorem that we need, the Product Theorem (3.4). It may be paraphrased as follows: If a compact 3-manifold $B$ has the same boundary as has $M \times I$, for $M$ a 2-manifold, if it has the same homotopy type as $M \times I$, and if the Poincaré conjecture is true

Received by the editors May 3, 1965 and, in revised form, January 28, 1966. 
inside $B$, then $B$ is homeomorphic to $M \times I$. Actually Theorem (3.4) is quite a bit stronger than this, and somewhat more useful. This is related to a result of John Stallings in [8].

In $\S \S 4-7$ we get necessary and sufficient conditions for unknotting in cases (i) through (iv) above.

In what follows a manifold is a combinatorial manifold with possibly vacuous boundary. It is equipped with a fixed triangulation. If $B$ is a manifold we denote its boundary by $\partial B$. All maps between manifolds are assumed to be piecewise linear.

\section{Definitions and preliminary propositions.}

Definition. A 3-manifold $B$ is said to be a Poincaré 3-manifold if every homotopy 3-cell in $B$ is homeomorphic to a 3-cell. A homotopy 3-cell in $B$ is a connected, simply connected, compact submanifold of $B$, bounded by a 2-sphere.

A word about Poincaré 3-manifolds. The Poincaré conjecture in dimension 3 is true if and only if every homotopy 3-cell is homeomorphic to a 3-cell. Thus a Poincaré 3-manifold is one in which the Poincaré conjecture is true. The extent of this notion is indicated by:

(2.1) Proposition. (a) Euclidean 3-space is a Poincaré 3-manifold.

(b) If $B$ is a Poincaré 3-manifold, so is every 3-dimensional submanifold of $B$.

(c) If $B$ is a Poincaré 3-manifold, and is a covering space of a 3-manifold $B^{\prime}$, then $B^{\prime}$ is a Poincaré 3-manifold.

(d) If $M$ is a 2-manifold then $M \times I$ is a Poincaré 3-manifold.

Proof. (a) This is Alexander's theorem.

(b) This is clear.

(c) Let $\pi: B \rightarrow B^{\prime}$ be a covering map, and let $C \subset B^{\prime}$ be a homotopy 3-cell. If $i^{\prime}: C \rightarrow B^{\prime}$ is the inclusion function, then there is a continuous function $i: C \rightarrow B$ so that $\pi i=i^{\prime}$, because $C$ is simply connected. Then $i$ is a homeomorphism. Since $B$ is a Poincaré 3-manifold, $i(C)$, and hence $C$, is homeomorphic to a 3-cell.

(d) We may assume $M$ is connected.

If $M$ is compact and orientable then $M \times I$ can be embedded in $R^{3}$, Euclidean 3-space, so (a) and (b) show that $M \times I$ is a Poincaré 3-manifold.

If $M$ is compact and nonorientable, then $M$ has an orientable double covering $N$, which is also compact. But $N \times I$ is a Poincaré 3-manifold and covers $M \times I$ so by (c), $M \times I$ is a Poincaré 3-manifold.

Finally suppose $M$ is noncompact, and let $C \subset M \times I$ be a homotopy 3-cell. Since $C$ is compact we can choose a compact submanifold $N$ of $M$ so that $C \subset N \times I$. By (b) again, $C$ is homeomorphic to a 3-cell. 
Definition. Let $N$ and $P$ be manifolds. An embedding $f: N \rightarrow P$ is said to be regular if

$$
f(\partial N)=f(N) \cap \partial P .
$$

That is, the image of $N$ meets the boundary of $P$ exactly in the boundary of $N$. Notice that if $N$ has no boundary, this means that $f(N)$ is contained in the interior of $P$. All our unknotting theorems are proved with respect to regular embeddings.

The Dehn lemma, as proved by Papakyriakopoulos, says that a simple closed curve in a 3-manifold, which is homotopic to a point, and which is contained in the boundary of the 3-manifold, bounds an embedded disk. When considering the fundamental group of a space, one usually considers homotopy classes of loops with fixed base point. However, one may equally well consider homotopy classes of paths with fixed end points. The only difference is that there is no preferred class to play the role of the point loop. Proposition (2.3) part (a) is the proof of the form that the Dehn lemma takes in this point of view. The extra difficulty which occurs because of the lack of a "trivial" path is taken care of in Lemma (2.2) part (a).

Shapiro and Whitehead on the other hand have proved a generalization of the Dehn lemma which says, in particular, that given two disjoint simple closed curves in the boundary of a 3-manifold, homotopic to one another, and neither null-homotopic and which "preserve orientation", then they bound an embedded annulus. Proposition (2.3) part (b) shows that the theorem is still valid if one relaxes the condition that both curves be simple, and only assumes that one of them is simple.

(2.2) Lemma. (a) Let $D$ be a 2-disk and let $p_{0}, p_{1} \in \partial D$. Let $K \subset D$ be a subcomplex which separates $p_{0}$ from $p_{1}$. Then $K$ contains a subcomplex which is a simple arc regularly embedded in $D$, and which also separates $p_{0}$ from $p_{1}$.

(b) Let $A$ be an annulus. Let $K \subset A$ be a subcomplex which separates the two components of $\partial A$. Then $K$ contains a subcomplex which is a simple loop regularly embedded in $A$, and which also separates the two components of $\partial A$.

Proof. In both cases let $L$ be a minimal subcomplex of $K$ which still separates. While it is intuitively clear that $L$ is the desired subcomplex, I cannot find a short proof of this fact. I list the steps of a long proof, each being verified by an appeal to the minimality of $L$.

1. $L$ contains no isolated vertices.

2. $L$ contains no 2-simplexes.

3. In case (a) $L$ contains no 1-simplex on $\partial D$.

4. No vertex of $L$ in the interior of $D$ (or $A$ ) is an end point of a single 1-simplex of $K$. 
5. $L$ contains no simple loops homotopic to a point in $D$ (or $A$ ).

6. Choose a vertex $v_{0}$ of $L$ which in case (a) is on $\partial D$. Pick an edge $e_{1}$ of $L$ having $v_{0}$ as a vertex, and let $v_{1}$ be the other vertex of $e_{1}$. Now pick an edge $e_{2} \neq e_{1}$ of $L$ having $e_{1}$ as vertex and continue.

In case (a) we get a new vertex and a new edge at each stage since $L$ contains no loops. This procedure only terminates when we reach $\partial D$ again, and it gives us a simple arc regularly embedded in $D$. By minimality of $L$, this arc separates $p_{0}$ from $p_{1}$.

In case (b) we must finally form a loop, which cannot be homotopic to a point. Such a loop separates the components of $\partial A$.

7. In either case the subcomplex constructed in 6 is all of $L$ since it separates.

(2.3) Proposition. Let $B$ be a 3-manifold, and $M$ a 2-dimensional submanifold of $\partial B$.

(a) Let $\alpha$ be a simple arc in $\partial B$ which meets $M$ exactly in its end points. Suppose $\alpha$ is homotopic in $B$, with end points fixed, to a path in $M$. Then there is a regularly embedded disk $D$ in $B$ whose boundary is $\alpha \cup(D \cap M)$, and $D \cap M$ is a regularly embedded arc in $M$.

(b) Let $\lambda$ be a simple loop in $\partial B$, not homotopic to a point in $B$, and not meeting $M$. Suppose $\lambda$ is freely homotopic to a loop in M. Suppose further that $\lambda$ generates an orientation preserving covering transformation in the universal cover of $B$ (we say $\lambda$ is an orientation preserving loop in $B$ ). Then there is a regularly embedded annulus $A$ in $B$ whose boundary is $\lambda \cup(A \cap M)$ and $A \cap M$ is regularly embedded in $M$.

Proof. Let $H=B \times\{0\} \cup M \times I \cup B \times\{1\}$. Then $H$ is a 3-manifold whose boundary is:

$$
(\partial B-M) \times\{0\} \cup(\partial M) \times I \cup(\partial B-M) \times\{1\} .
$$

(a) Since $\alpha$ is homotopic with end points fixed to a path in $M$, the simple loop

$$
\alpha \times\{0\} \cup(\partial \alpha) \times I \cup \alpha \times\{1\}
$$

in $\partial H$ is homotopic to a point in $H$. By Dehn's lemma [6] it bounds a nonsingular disk $E$ regularly embedded in $H$. Let $p_{0}, p_{1}$ be the midpoints of $\alpha \times\{0\}$ and $\alpha \times\{1\}$ respectively. Let $K=E \cap M \times\{0\}$. Then $K$ separates $p_{0}$ from $p_{1}$ in $E$, so by part (a) of Lemma (2.2) we can find a simple $\operatorname{arc} \beta \times\{0\} \subset K$, regularly embedded in $E$ which also separates $p_{0}$ from $p_{1}$ in $E$. Then $\beta \times\{0\}$ is regularly embedded in $M \times\{0\}$, and has the same end points as $\alpha \times\{0\}$. The simple loop $(\alpha \cup \beta) \times\{0\}$ is homotopic to a point in $H$. But the composition

$$
\pi_{1}(B) \rightarrow \pi_{1}(B \times 0) \rightarrow \pi_{1}(H) \rightarrow \pi_{1}(B \times I)
$$

is a monomorphism, so $\alpha \cup \beta$ is homotopic to a point in $B$. By Dehn's lemma again it bounds a nonsingular disk $D$ regularly embedded in $B$. 
(b) The proof is similar to (a), we use part (b) of Lemma (2.2), and the generalization of Dehn's lemma due to Shapiro and Whitehead [7]. It is to apply this last theorem that we need the special assumptions about $\lambda$.

The next proposition establishes the existence of a tubular neighborhood for a 2-manifold, regularly embedded in a 3-manifold. One could prove this for a regularly embedded $(n-1)$-manifold in an $n$-manifold. There is a difficulty with the "cutting apart" operation however. In three dimensions topological, triangulated, combinatorial, etc., manifolds are all the same. In higher dimensions the situation is not at all clear. In the "cutting apart" operation one may go from a combinatiorial manifold to the possibly weaker, $n$-star manifold. For this reason we have restricted the dimensions.

(2.4) Proposition. Let $B$ be a compact 3-manifold, and $M$ a regularly embedded compact 2-dimensional submanifold of $B$. Then $M$ has a tubular neighborhood in B. That is, a neighborhood $U$ such that $U$ is homeomorphic to a 1-dimensional disk bundle over $M$, the homeomorphism carrying $M$ to the zero section of the bundle, and carryng $U \cap \partial B$ to the part of the bundle over $\partial M$.

Proof. We cut $B$ apart along $M$. Since we have restricted ourselves to three dimensions, we need not worry about what type of manifold we get by this process. We may proceed as follows; each closed 2-simplex of $M$ is a face of exactly two 3-simplexes of $B$. Replace the closed 2-simplex by two copies of itself, one on each of the 3-simplexes. The resulting space is easily seen to be a manifold, it is necessarily a combinatorial manifold. This gives us a compact 3-manifold $\widetilde{B}$. $M$ goes into $\tilde{M} \subset \partial \widetilde{B}$, a double covering of $M$. The second regular neighborhood $V$ of $\tilde{M}$ in $\tilde{B}$ is homeomorphic to $\tilde{M} \times I$ according to Whitehead's regular neighborhood theory [9]. Such a homeomorphism may be assumed to carry $V \cap(\partial \widetilde{B}-\tilde{M})$ to $(\partial \tilde{M}) \times(0,1)$. When we paste $\widetilde{B}$ back together to get $B$, then $V$ becomes the desired neighborhood $U$.

In what follows we shall make constant use of Van Kampen's theorem (as in [5, p. 243]). A little notation here will be useful. For groups $G_{1}, G_{2}$ and $U$ and monomorphisms $i_{r}: U \rightarrow G_{r}, r=1,2$ we denote by $G_{1} *_{U} G_{2}$ the free product of $G_{1}$ and $G_{2}$ with amalgamation of the group $U$ via $i_{1}$ and $i_{2}$. That is $G_{1} *_{U} G_{2}$ is generated by the union of a set of generators of $G_{1}$ with a set of generators of $G_{2}$, and has as relations those of $G_{1}$, those of $G_{2}$, and those of the form $i_{1}(u)=i_{2}(u)$ for each $u$ in $U$. Now $G_{1} *_{U} G_{2}$ depends on $i_{1}$ and $i_{2}$. But for us $i_{1}$ and $i_{2}$ will be maps of fundamental groups induced by inclusion functions. Since $i_{1}$ and $i_{2}$ are fixed we may regard $U$ as the intersection of the groups $G_{1}$ and $G_{2}$. We shall do this in the future without further comment.

The following theorem is from $[4$, p. 314$]$.

THEOREM. Let $G_{1}, G_{2}$ and $U$ be as above. Let $x_{i k}$ be a fixed choice of coset 
representatives of the right cosets of $U$ in $G_{i}$ different from $U$ itself. Then each element of $G_{1} *_{U} G_{2}$ has a unique representative of the form $u z_{1} \cdots z_{m}$ where $u \in U$, the $z_{i}$ are from among the $x_{i k}$, and $z_{j}$ and $z_{j+1}$ come from different G's.

We draw a useful corollary from this:

(2.5) Proposition. Let $G_{r}, r=1,2$ be groups with intersection the common subgroup $U$. Let $H_{r}, r=1,2$ be a subgroup of $G_{r}$, which also contains $U$. If the natural homomorphism

$$
j: H_{1} *_{U} H_{2} \rightarrow G_{1} *{ }_{U} G_{2}
$$

is an isomorphism then $H_{r}=G_{r}$.

Proof. Let $i_{1}: G_{1} \rightarrow G_{1} *{ }_{U} G_{2}$ be the natural monomorphism. Let $g \in G_{1}$ and write $g=u x_{1, k}$ for some $u \in U$ where $x_{i k}$ are as in the preceding theorem. Using a subset of the $x_{i k}$ to represent the cosets of $U$ in $H_{i}$, by the preceding theorem there is a unique representative $u^{\prime} z_{1} \cdots z_{m}$ of the element of $H_{1} *_{U} H_{2}$ which $j$ carries into $i_{1}(g)$. Then $u^{\prime} z_{1} \cdots z_{m}$ and $u x_{1, k}$ both represent $i_{1}(g)$ in $G_{1} *_{U} G_{2}$. Hence $u=u^{\prime}, z_{1}=x_{1, k}$ and $m=1$. But then $x_{1, k}$ is a representative of a coset of $U$ in $H_{1}$, so $g=u x_{i, k}$ is in $H_{1}$. Since $g$ was arbitrary $H_{1}=G_{1}$. Similarly $H_{2}=G_{2}$.

\section{The Product Theorem.}

(3.1) Theorem. Let $B$ be a compact, connected, Poincaré 3-manifold. Let $M$ be a compact connected 2-manifold other than the projective plane. Let

$$
h: M \times\{0\} \cup(\partial M) \times I \rightarrow \partial B
$$

be an embedding such that:

(1) $M_{1}=\partial B-h(M \times\{0\} \cup(\partial M) \times[0,1))$ is a nonvacuous, connected submanifold of $\partial B$.

(2) For $i=0,1$ the natural homomorphisms

$$
\pi_{1}\left(M_{i}\right) \rightarrow \pi_{1}(B)
$$

are isomorphisms, where $M_{0}=h(M \times\{0\})$. Then $h$ can be extended to a homeomorphism of $M \times I$ onto $B$.

Proof. The proof is in three steps. In Step (I) we reduce by an induction argument to the case where $M$ has at most one handle or one cross-cap. In Step (II) we reduce the theorem for manifolds with boundary to the theorem for a disk. In Step (III) we verify the theorem for the few special cases left.

Step (I). Let $c(M)$, the complexity of $M$, be the number of handles of $M$ if $M$ is orientable, or the number of cross-caps of $M$ if $M$ is nonorientable. Assume $c(M) \geqq 2$. We shall split $M$ into two pieces of lower complexity, and also split 
$B$. We then show that the theorem for these simpler manifolds implies the theorem for $M$.

As for the splitting, we may choose a simple loop $\mu$ regularly embedded in $M$ which splits $M$ into two submanifolds $N$ and $P$. Choose $\mu$ so that $c(N)>0$ and $c(P)>0$. Then $c(N)+c(P) \leqq c(M)$, so each of $c(N)$ and $c(P)$ is less than $c(M)$.

Let $\lambda=h(\mu \times\{0\})$. Then $\lambda$ does not meet $M_{1}$, but $\lambda$ is homotopic to a loop in $M_{1}$ since $\pi_{1}\left(M_{1}\right) \rightarrow \pi_{1}(B)$ is onto. Moreover $\lambda$ is not homotopic to a point in $M_{0}$, and since $\pi_{1}\left(M_{0}\right) \rightarrow \pi_{1}(B)$ is a monomorphism, $\lambda$ is not homotopic to a point in $B$. We wish to apply Proposition (2.3) part (b) to $\lambda$ and $M_{1}$. For this we need $\lambda$ to be an orientable loop in $B$. But $\lambda$ is an orientable loop in $M_{0}$ since it separates $M_{0}$. From the fact that $M_{0}$ lies on the boundary of $B$ we conclude that $\lambda$ is an orientable loop in $B$. Thus Proposition (2.3) part (b) can be applied.

Let $A$ be a regularly embedded annulus in $B$ with one boundary component $\lambda$ and the other in the interior of $M_{1}$. Extend $h$ to a homeorecrphism of $\mu \times I$ onto $A$.

We now claim that $A$ splits $B$ into two pieces. Suppose to the contrary that $B-A$ is connected. Choose a path in $B-A$ joining points in distinct components of $M_{0}-\lambda$. Since $\pi_{1}\left(M_{0}\right) \rightarrow \pi_{1}(B)$ is onto we can deform our path, holding its end points fixed, to a path in $M_{0}$. Now before the deformation our path meets $A$ an even number, (in fact zero) of times. Thus after the deformation we have a path whose mod 2 intersection number with $A$ is zero. This means a path which starts in one component of $M_{0}-\lambda$, intersects $\lambda$ an even number of times, and ends in the other component of $M_{0}-\lambda$. This is clearly impossible.

Let $C$ and $D$ be the closures of the two components of $B-A$, notation chosen so that

$$
\begin{aligned}
& C \supset h(N \times 0)=N_{0}, \\
& D \supset h(P \times 0)=P_{0} .
\end{aligned}
$$

We shall be finished with Step (I) when we prove:

(3.2) LEMMA. Under the hypotheses of Theorem (3.1) assume $h$ has been extended to a regular embedding of $\mu \times I$ into $B$ where $\mu$ is a simple separating loop in the interior of $M$ not homotopic to a point. Assume further that $h(\mu \times\{1\})$ is regularly embedded in $M_{1}$. With notation as above we let

$$
\begin{aligned}
& k=h \mid N \times\{0\} \cup(\partial N) \times I, \\
& l=h \mid P \times\{0\} \cup(\partial P) \times I .
\end{aligned}
$$

Then the triples $(C, N, k)$ and $(D, P, l)$ satisfy all hypotheses of Theorem (3.1). Extensions of $k$ and $l$ give an extension of $h$, 
Notice that for this lemma we do not assume that $N$ and $P$ have lower complexity than $M$ or that $c(M) \geqq 2$.

Proof of (3.2). Clearly $C$ and $D$ are compact, and connected. Since they are submanifold of $B$, Proposition (2.1) says that they are Poincare manifolds.

Now $N_{1}=C \cap M_{1}$ and $P_{1}=D \cap M_{1}$ are the closures of the components of $M_{1}-h(\mu \times\{1\}$. Thus they are nonvacuous and connected. This establishes hypothesis (1).

We turn to the algebraic hypothesis (2). We wish to apply Proposition (2.5), and for this we must show that certain homomorphisms are monomorphisms. First $\pi_{1}(\lambda) \rightarrow \pi_{1}\left(N_{0}\right)$ and $\pi_{1}(\lambda) \rightarrow \pi_{1}\left(P_{0}\right)$ are monomorphisms since $\lambda$ is a boundary component of both $N_{0}$ and $P_{0}$, and neither is a disk. Thus $\pi_{1}\left(N_{0}\right) *_{\pi_{1}(\lambda)} \pi_{1}\left(P_{0}\right)$ is well defined and by Van Kampen's theorem is isomorphic to $\pi_{1}\left(M_{0}\right)$. Second, using that $\pi_{1}(\lambda) \rightarrow \pi_{1}(A)$ and $\pi_{1}\left(M_{0}\right) \rightarrow \pi_{1}(B)$ are isomorphisms it is not hard to check that $\pi_{1}(A) \rightarrow \pi_{1}(C)$ and $\pi_{1}(A) \rightarrow \pi_{1}(D)$ are monomorphisms also. Thus $\pi_{1}(C) *_{\pi_{1}(A)} \pi_{1}(D)$ is well defined and is isomorphic to $\pi_{1}(B)$. In the commutative diagram

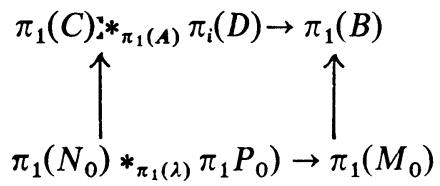

Three of the maps are isomorphisms, thus the left-hand vertical map is also an isomorphism. By Proposition (2.5) now, $\pi_{1}\left(N_{0}\right) \rightarrow \pi_{1}(C)$ and $\pi_{1}\left(P_{0}\right) \rightarrow \pi_{1}(D)$ are isomorphisms. Similarly $\pi_{1}\left(N_{1}\right) \rightarrow \pi_{1}(C)$ and $\pi_{1}\left(P_{1}\right) \rightarrow \pi_{1}(D)$ are isomorphisms. This completes the proof of Lemma (3.2), and of Step(I). Thus we have reduced the theorem for manifolds with complexity at least two, to the theorem for manifolds with complexity at most one and with nonvacuous boundary.

Step (II). In this step we reduce the theorem for manifolds with boundary to the theorem for the disk. The idea is similar to Step (I), only we split $B$ along a nonseparating disk instead of a separating annulus.

Let $\alpha$ be a simple arc, regularly embedded in $M$, and not separating $M$. Now if $\alpha$ joins points of distinct boundary components, then cutting $M$ apart along $\alpha$ decreases the number of boundary components by one and does not alter the complexity. On the other hand if $\alpha$ joins points of the same boundary component, then cutting $M$ apart along $\alpha$ decreases the complexity by one, and increases the number of boundary components by at most one. Thus we shall do an induction argument on twice the complexity plus the number of boundary components.

Since $\pi_{1}\left(M_{1}\right) \rightarrow \pi_{1}(B)$ is onto, the simple arc $h(\alpha \times\{0\} \cup(\partial \alpha) \times I)$ is homotopic with end points fixed to a path in $M_{1}$. By Proposition (2.3) part (a) we can extend $h$ to a regular embedding of $\alpha \times I$ into $B$ such that $h \mid \alpha \times\{1\}$ is a regular embedding in $M_{1}$. Denote by $D$ the image $h(\alpha \times I)$. 
Choose a tubular neighborhood $U$ of $D$ in $B$ (Proposition (2.4)). Since $D$ is contractible, $U$ is equivalent to $D \times[-1,1]$ and $U \cap M_{0}$ is equivalent to $h(\alpha \times\{0\}) \times[-1,1]$. Let $V$ be the neighborhood of $\alpha$ in $M$ such that $h(V \times\{0\})=U \cap M_{0}$. Now $h$ has already been defined on $V \times\{0\} \cup \alpha \times I$, and it is easy to see that $h$ can be extended to a homeomorphism of $V \times I$ onto $U$.

Let $U^{0}$ be the interior of $U$ in $B$, and let $V^{0}$ be the interior of $V$ in $M$. We may assume $U^{0}=h\left(V^{0} \times I\right)$. Let $B^{\prime}=B-U^{0}, M^{\prime}=M-V^{0}$ and $h^{\prime}=h \mid M^{\prime} \times\{0\} \cup\left(\partial M^{\prime}\right) \times I$. We shall have completed Step (II) when we show:

(3.3) Lemma. Under the hypotheses of Theorem (3.1), and with a nonseparating arc $\alpha$, regularly embedded in $M$, suppose an extension of $h$ over $\alpha \times I$ is given so that $h \mid \alpha \times\{1\}$ is a regular embedding in $M_{1}$. With notation as above choose an extension over $V \times I$ to $U$. Then the triple $\left(B^{\prime}, M^{\prime}, h^{\prime}\right)$ satisfies all hypotheses of Theorem (3.1).

This lemma, and Lemma (3.2) are singled out since they will be used again later.

Proof of (3.3). First note that $B^{\prime}$ is a compact Poincaré manifold since it is a closed submanifold of $B$. Using the fact that $M^{\prime}$ and $B$ are connected it is not hard to show that $B^{\prime}$ is connected. Now $M_{1}^{\prime}=B^{\prime} \cap M_{1}$ is nonvacuous, an argument as in Step (I) about intersection numbers shows that $M_{1}^{\prime}$ is connected. This verifies hypothesis (1).

To show that $\pi_{1}\left(M_{j}^{\prime}\right) \rightarrow \pi_{1}\left(B^{\prime}\right)$ is an isomorphism for $j=0,1$ we shall show that there is a retraction of $B$ onto $B^{\prime}$ which carries $M_{j}$ onto $M_{j}^{\prime}$. Then the isomorphism conclusion follows easily. We shall work on $V \times I$ and follow by $h$.

First retract $V \times I$ to $V \times\{0\} \cup\left(V-V^{0}\right) \times I$. Holding $\left(V-V^{0}\right) \times I$ fixed, retract $V \times\{0\}$ into an arc joining points in the distinct components of $\left(V-V^{0}\right) \times I$. Finish the retraction by mapping this arc into $M^{\prime} \times\{0\}$ holding its end points fixed. This is possible since $M^{\prime}$ is connected. See Figure 1.
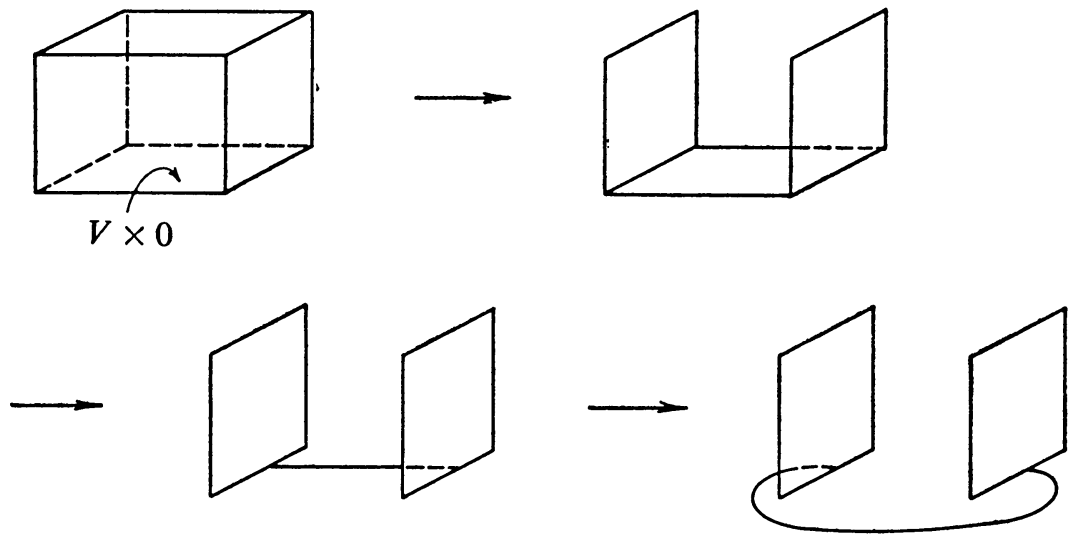

FIGURE 1 
Following this composed retraction by $h$ we get a retraction of $B$ to $B^{\prime}$, which carries $M_{0}$ to $M_{0}^{\prime}$. Of course, all this could have been done in $U$. A similar argument in $U$ retracts $B$ to $B^{\prime}$ carrying $M_{1}$ to $M_{1}^{\prime}$. This concludes the verifications of hypothesis (2) and of (3.3).

Step (III). In Steps (I) and (II) we have reduced the theorem for manifolds of complexity at least two, or with nonempty boundary, to the theorem for the disk. We shall have completed the proof when we prove the theorem for the disk, the two-sphere, and the torus.

The disk. If $M$ is a disk then $\pi_{1}(M) \simeq \pi_{1}\left(M_{0}\right) \simeq \pi_{1}(B) \simeq \pi_{1}\left(M_{1}\right) \simeq 1$. We can extend $h$ to a homeomorphism of $M \times 1$ onto $M_{1}$, say radially from the center of the disks. Now $B$ is compact, connected, simply connected, and $h$ maps $\partial(M \times I)$ homeomorphically onto $\partial B$ which is thus a 2 -sphere. Since $B$ is a Poincaré manifold, it is a 3-cell. Thus we can extend $h$, say radially again over $M \times I$.

The 2-sphere. Since $\partial M=\varnothing$ we must only show that $B$ is homeomorphic to $M \times I$. For if $H$ is any homeomorphism of $M \times I$ onto $B$, by reflecting in $M \times 1 / 2$, if necessary, we may assume that $h(M \times 0)=H(M \times 0)$. Letting $h_{1}=H \mid M \times 0$ the map $H\left(h_{1}^{-1} h \times i d\right)$ is the desired extension of $h$.

Form a new manifold $B_{1}$ by attaching a 3-cell $C$ onto $B$ along the 2-sphere $M_{0}$. Now $\partial B_{1}=M_{1}$ which is a 2-sphere since it is closed and simply connected. Furthermore, $B_{1}$ is compact, connected, and simply connected by Van Kampen's theorem. If we can show that $B_{1}$ is a Poincaré manifold, then we will know that it is a 3-cell. To see this we shall embed $B_{1}$ in $B$.

We note that there is a homeomorphic image, $B_{1}^{\prime}$, of $B_{1}$ contained in the interior of $B_{1}$ (using a collar neighborhood of $\partial B_{1}$ ). Choose a 3-cell in the interior of $B$, not meeting $B_{1}^{\prime}$. Map this 3-cell by an orientation preserving map homeomorphically onto $C$. According to Gugenheim [3], we can extend to a homeomorphism of $B_{1}$ onto itself. Since the image of $B_{1}^{\prime}$ under this homeomorphism misses $C$, it is contained in $B$. Thus $B_{1}$ is a 3-cell.

Hence we can map $B_{1}$ homeomorphically onto the set of vectors in $R^{3}$ of length not greater than 1 , and using Gugenheim again, we may insist that $C$ go onto the set of vectors of length not exceeding $1 / 2$. This maps $B$ homeomorphically onto $M \times I$.

The torus. For this case we put together the methods of Steps (I) and (II).

Choose a simple loop in $M$ not homotopic to a point. We can apply Proposition (2.3) part (b) since $M_{0}$, and hence $B$, is orientable. This gives us an annulus in $B$ whose ends are simple loops in $M_{0}$ and $M_{1}$ not homotopic to a point. A tubular neighborhood of this annulus meets $M_{0}$ and $M_{1}$ in annuli. We may retract this tubular neighborhood onto its sides and its intersection with $M_{0}$. Then we may take the annulus in $M_{0}$ and retract it onto its complement which is also an annulus. As in Step (II) this verifies the algebraic hypothesis (2) for the complement of the interior of our tubular neighborhood. 
The other hypotheses are easily verified, and this reduces the case for the torus to the case for the annulus. This finishes the proof of Theorem (3.1).

We get a generalization of this theorem by applying Theorem (1.1) of [1].

(3.4) Theorem. Let $B$ be a compact connected Poincaré 3-manifold. Let $M$ be a compact connected 2-manifold not the projective plane such that $\chi(M) \geqq \chi(B)$ where $\chi$ is the Euler characteristic. Let

$$
h: M \times\{0\} \cup(\partial M) \times I \rightarrow \partial B
$$

be an embedding to a proper subset of $\partial B$. Suppose that the natural, homomorphism

$$
\pi_{1}(h(M \times\{0\})) \rightarrow \pi_{1}(B)
$$

is onto. Then $h$ can be extended to a homeomorphism of $M \times I$ onto $B$.

Before we proced to the proof of this theorem let us make a few remarks about what it says. The manifold $B$ is a cobordism between $M$ and

$$
N=\partial B-h(\text { int } M \times\{0\}) .
$$

The theorem says that under certain mild hypotheses, this cobordism is a cartesian product of $M$ with an interval. Mazur has shown that if the natural maps of $M$ and $N$ into $B$ are simple homotopy equivalences (i.e. $B$ is an $s$-cobordism), then $B$ is a cartesian product of $M$ with an interval, in those dimensions where the Poincare conjecture is known to be true. It is also known that if the natural maps are only homotopy equivalences, then $B$ need not be a product space. Our theorem says that in three dimensions we need not even assume that both maps are homotopy equivalences.

Proof of the theorem. Let $T=\mathrm{Cl}(\partial B-h(M \times\{0\}))$. Then applying Theorem 1.1 of [1] to $B$ and $S=h(M \times\{0\})$ we have that both $S$ and $T$ are strong deformation retracts of $B$. But $h((\partial M) \times I)$ has $h((\partial M) \times\{1\})$ as a strong deformation rectract, so $T$ has

$$
M_{1}=\partial B-h(M \times\{0\} \cup(\partial M) \times[0,1))
$$

as strong deformation retract. In particular the natural homomorphisms

$$
\pi_{1}\left(M_{i}\right) \rightarrow \pi_{1}(B), \quad i=0,1,
$$

are isomorphisms, where $M_{0}=h(M \times\{0\})$. By Theorem (3.1) we are finished.

4. The unknotting of disks. In this section we shall consider embeddings of a disk in $M^{2} \times I$. The "natural" or unknotted positions will be $\alpha \times I$ for $\alpha$ an arc in $M$. We shall consider the domain disk to be $I \times I$ and we shall ask to unknot embeddings $f$ such that $f(s, 0)=(\alpha(s), 0)$. If $\alpha$ is contained in the interior of $M$, then to all intents and purposes it is a point path, and we are 
asking about the unknotting of arcs in $M \times I$. This is treated separately in $\S 6$, so here we make the restriction that $\alpha$ be regularly embedded in $M$. With $\alpha$ regularly embedded in $M$, we have $\alpha \times I$ a regularly embedded disk in $M \times I$. Since a homeomorphism of $M \times I$ to itself carries regular embeddings to regular embeddings, we must require that $f$ be a regular embedding. We shall also require that $f(0, s)=(\alpha(0), s)$ and $f(1, s)=(\alpha(1), s)$ but this is no real restriction. For consider a collar neighborhood of $\partial M \times 0$ in $M \times 0$. There is a homeomorphism of $M \times I$ onto itself which carries this collar neighborhood onto $(\partial M) \times I$. Since we required $f(x, 0)=(\alpha(s), 0)$, this "straightens out the edges", of $f(I \times I)$.

(4.1) THEOREM. Let $\alpha_{j}, j=1, \cdots, m$, be a finite collection of pairwise disjoint arcs regularly embedded in the compact 2-manifold $M$. Let $f$ be a regular embedding of $\bigcup_{j=1}^{m} I \times I \times\{j\}$ in $M \times I$ such that

$$
\begin{aligned}
& f(s, o, j)=\left(\alpha_{j}(s), o\right), \\
& f(o, s, j)=\left(\alpha_{j}(o), s\right), \\
& f(1, s, j)=\left(\alpha_{j}(1), s\right),
\end{aligned}
$$

for $j=1, \cdots, m$, and such that $f \mid \bigcup_{j=1}^{m} I \times\{1\} \times\{j\}$ is a regular embedding in $M \times\{1\}$. Then there exists a homeomorphism $h$ of $M \times I$ onto itself such that

$$
\begin{aligned}
h(m, o) & =(m, o), & m \in M, \\
h(m, t) & =(m, t), & m \in \partial M, t \in I, \\
h f(s, t, j) & =\left(\alpha_{j}(s), t\right), & j=1, \cdots, m .
\end{aligned}
$$

Proof. Since we can work with one component at a time it is clearly no loss of generality to assume that $M$ is connected. We proceed by induction on $m$, so assume first that $m=1$. We construct $h^{-1}$ rather than $h$. Let

$$
k:(M \times\{o\}) \cup((\partial M) \times I) \cup\left(\alpha_{1}(I) \times I\right) \rightarrow M \times I
$$

be defined by

$$
\begin{aligned}
k(m, o) & =(m, o), & m \in M, \\
k(m, t) & =(m, t), & m \in \partial M, t \in I, \\
k\left(\alpha_{1}(s), t\right) & =f(s, t), & s \in I, t \in I .
\end{aligned}
$$

We shall extend $k$ to a homeomorphism of $M \times I$ onto itself, whence $h=k^{-1}$ satisfies all conditions of the theorem.

There are two cases according as $\alpha_{1}$ does or does not separate $M$.

Case I. $M-\alpha_{1}$ is connected. We note that $M \times I$ is a Poincaré manifold by (2.1). And if we take for $B$ the manifold $M \times I$ then the pair $B . k \mid M \times 0 \cup(\partial M) \times I$ satisfies all hypotheses of Theorem (3.1). Then by Lemma (3.3) and Theorem (3.1) we can extend $k$ to a homeomorphism of $M \times I$ onto itself. 
Case II. $M-\alpha_{1}$ is not connected. Let the closures of the two components of $M-\alpha_{1}$ be denoted $M^{\prime}$ and $M^{\prime \prime}$. Now by the simple intersection number argument which appears in the proof of Case $I$ of (3.1) we can see that $M \times I$ $-f(I \times I)$ is not connected. Let the closures of the two components be denoted $B^{\prime}, B^{\prime \prime}$, with notation chosen so that $B^{\prime} \supset M^{\prime}$.

In the commutative diagram (the isomorphisms are by Van Kampen's theorem)

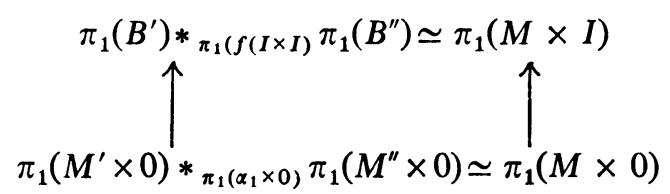

the right-hand vertical map is an isomorphism. Thus the left-hand vertical map is also. By (2.5) both

$$
\pi_{1}\left(M^{\prime}\right) \rightarrow \pi_{1}\left(B^{\prime}\right) \text { and } \pi_{1}\left(M^{\prime \prime}\right) \rightarrow \pi_{1}\left(B^{\prime \prime}\right)
$$

are isomorphisms, in particular they are onto. Now

$$
\begin{aligned}
& \chi(M \times I)=\chi\left(B^{\prime}\right)+\chi\left(B^{\prime \prime}\right)-\chi(f(I \times I))=\chi\left(B^{\prime}\right)+\chi\left(B^{\prime \prime}\right)-1 . \\
& \chi(M \times 0)=\chi\left(M^{\prime} \times 0\right)+\chi\left(M^{\prime \prime} \times 0\right)-\chi\left(\alpha_{1} \times 0\right)=\chi\left(M^{\prime}\right)+\chi\left(M^{\prime \prime}\right)-1,
\end{aligned}
$$

Since $\chi(M \times 0)=\chi(M \times I)$ we may assume that $\chi\left(M^{\prime}\right) \geqq \chi\left(B^{\prime}\right)$ (otherwise $\left.\chi\left(M^{\prime \prime}\right)>\chi\left(B^{\prime \prime}\right)\right)$. According to (3.4) we can extend $k \mid M^{\prime} \times 0 \cup\left(\partial M^{\prime}\right) \times I$ to a homeomorphism of $M^{\prime} \times I$ onto $B^{\prime}$. In particular $\chi\left(M^{\prime}\right)=\chi\left(B^{\prime}\right)$, so $\chi\left(M^{\prime \prime}\right)=\chi\left(B^{\prime \prime}\right)$ and we may use (3.4) again to extend $k \mid M^{\prime \prime} \times 0 \cup\left(\partial M^{\prime \prime}\right) \times I$ to a homeomorphism of $M^{\prime \prime} \times I$ onto $B^{\prime \prime}$. These two extensions together extend $k$ to a homeomorphism of $M \times I$ onto itself as desired.

This finishes the case $m=1$, we assume inductively that $m>1$, and that the theorem has been proved for $m-1$ arcs.

Let $h_{1}$ be a homeomorphism of $M \times I$ onto itself which is the identity in $M \times 0 \cup(\partial M) \times I$ and such that $h_{1} f(s, t, 1)=\left(\alpha_{1}(s), t\right)$. Let $V$ be a tubular neighborhood of $\alpha_{1}(\mathrm{I})$ in $M$, so small that

$$
(V \times I) \cap h_{1} f(I \times I \times\{j\})=\varnothing \text { for } j>1 \text {. }
$$

Let $V^{0}$ be the interior of $V$ in $M$ and let $M_{1}=M-V^{0}$, a compact 2-manifold Let

$$
f_{1}: \bigcup_{j=2}^{m} I \times I \times\{j\} \rightarrow M_{1} \times I
$$

be defined by $f_{1}=h_{1} f$. Then the pair $f_{1}, M_{1}$ satisfies all hypotheses of this theorem, but with $m-1$ arcs. Let $h_{2}$ be the homeomorphism of $M_{1} \times I$ onto itself guaranteed by the induction assumption. Since $h_{2} \mid\left(\partial M_{1}\right) \times I$ is the identity map, we can extend $h_{2}$ over $V \times I$ by making it the identity. Then $h=h_{2} h_{1}$ is easily seen to satisfy all conditions of the theorem. 
5. The unknotting of annuli. In this section we shall consider the unknotting of a regular embedding $f$ of $S^{1} \times I$ into $M \times I$, "based" at a simple loop $\lambda$ regularly embedded in $M$. By this we mean $f(s, o)=(\lambda(s), o)$ for $s \in S^{1}$, and $f \mid S^{1} \times\{1\}$ is a regular embedding in $M \times 1$. This second requirement is not essential, as in the last section, an argument involving a collar neighborhood of $\partial M \times 0$ shows this. We shall further require that $\lambda$ not be homotopic to a point. If $\lambda$ is homotopic to a point, it turns out that $f$ is the boundary of a tubular neighborhood of an embedded arc. This fact, which is demonstrated in $\$ 6$, enables us to handle the null-homotopic case the same way we handle an arc, and we do so in $\$ 6$.

We comment now on the structure of the proof. If $\lambda$ separates $M$, the unknotting becomes an easy application of (3.2). If $M$ is orientable, then $\lambda$ separates a neighborhood of itself in $M$, even though it does not separate $M$. In this case we find a handle in $M$ which contains $\lambda$. We " "unknot" the product of the handle with an interval in $M \times I$, and then working inside this smaller product, prove the special case which results there. An induction argument then extends our result to the case of finitely many annuli when $M$ is orientable. We next take up the case when $M$ is not orientable. In the orientable double covering of $M$, the loop $\lambda$ becomes either one or two loops. By the orientable case for finitely many loops we can use the theorem here to establish certain facts about the homotopy of $M$, which together with (3.4) finishing this case. An easy induction argument extends the result to the unknotting of a finite number of disks and annuli in an arbitrary $M$.

(5.1) THEOREM. Let $M$ be a compact orientable 2-manifold. Let $\lambda_{j}, j=1, \cdots, m$, be a finite number of pairwise disjoint, regularly embedded simple loops in $M$, none of which is null homotopic. Let

$$
f: \bigcup_{j=1}^{m} S^{1} \times I \times\{j\} \rightarrow M \times I
$$

be a regular embedding such that

$$
\begin{array}{ll}
f(s, 0, j)=\left(\lambda_{j}(s), 0\right), & s \in S^{\mathbf{1},} \\
f(s, 1, j) \in \operatorname{int} M \times\{1\}, & s \in S^{\mathbf{1}} .
\end{array}
$$

Then there exists a homeomorphism $h$ of $M \times I$ onto itself such that

$$
\begin{array}{rlr}
h(m, o) & =(m, o), & m \in M, \\
h(m, t) & =(m, t), & m \in \partial M, \quad t \in I, \\
h f(s, t, j) & =\left(\lambda_{j}(s), t\right) & s \in S^{1}, \quad t \in I .
\end{array}
$$

Proof. We first remark that we may assume $M$ connected. The proof now proceeds by induction on $m$. We dispose of the reduction step of the induction 
first since it is almost identical to that of (4.1). The only difference is that when we remove a tubular neighborhood of $\lambda_{1}\left(S^{1}\right)$ to get $M_{1}$, we must check that no $\lambda_{j} j>1$ becomes null-homotopic. But this is immediate since $M_{1} \subset M$ and none is null-homotopic in $M$.

We may assume now that $m=1$ and $\lambda=\lambda_{1}$ is a single loop. There are two cases according as $\lambda$ does, or does not separate $M$.

Case I. $\lambda$ separates $M$. We define

$$
k: M \times\{0\} \cup(\partial M) \times I \cup \lambda\left(S^{1}\right) \times I \rightarrow M \times I
$$

by

$$
\begin{aligned}
k \mid M \times\{0\} \cup(\partial M) \times I & =\text { identity, } \\
k(\lambda(s), t) & =f(s, t), \quad s \in S^{\mathbf{1}}, \quad t \in I .
\end{aligned}
$$

As before we extend $k$ to a homeomorphism of $M \times I$ onto itself and take $h=k^{-1}$.

We first extend $k$ to a tubular neighborhood of $\lambda \times I$. Then by Lemma (3.2) and Theorem (3.1) we can further extend $k$ to a homeomorphism of $M \times I$ onto itself.

Case II. $\lambda$ does not separate $M$. Define $h$ to be as required on

$$
M \times\{0\} \cup(\partial M) \times I \cup f\left(S^{1} \times I\right) .
$$

If $M$ is a torus then $h$ can be extended to a homeomorphism of $M \times I$ onto itself by Case III of (3.1) for the torus. We may then assume that $M$ is not a torus. We claim that there is a simple separating loop $\delta$ in $M$, such that the component of $M-\delta$ which contains $\lambda$ is a torus with a hole in it; see Figure 2. For a tubular neighborhood of $\lambda$ in $M$ is an annulus by orientability, and we can choose an arc in the complement of the tubular neighborhood which joins the two components of its boundary. Thickening the arc to a band gives us, using orientability again, the torus with a hole whose boundary is $\delta$.

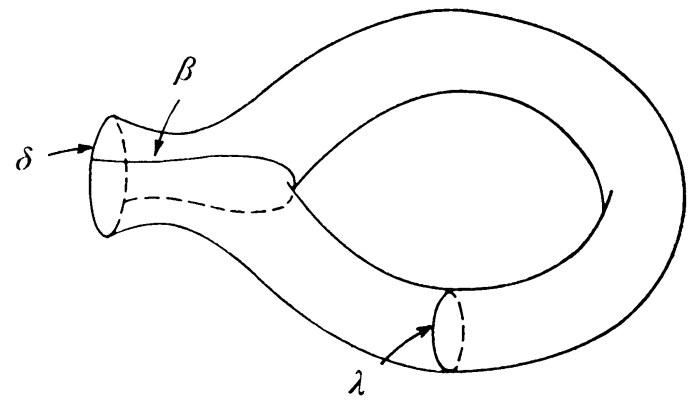

FIGURE 2

By (2.3) we can find an annulus $A$ regularly embedded in $M \times I$ such that $\partial A=\delta \times 0 \cup(A \cap(M \times 1))$ and $A \cap(M \times 1)$ is regularly embedded in $M \times 1$. For this we need that $\delta$ is not homotopic to a point, i.e. $M$ is not a torus. We 
claim that $A$ can be chosen disjoint from $f\left(S^{1} \times I\right)$. We first take $A$ to be in general position with respect to $f\left(S^{1} \times I\right)$. Then $A \cap f\left(S^{1} \times I\right)$ is a regularly embedded 1-dimensional submanifold of both $A$ and $f\left(S^{1} \times I\right)$. Thus $A \cap f\left(S^{1} \times I\right)$ is a finite number of disjoint simple closed curves regularly embedded in both, together with a finite number of disjoint simple arcs with their end points in $M_{1}$, (in $M_{1}$ since $\lambda \cap \delta=\varnothing$ ). Let $\mu$ be one of the simple loops. If $\mu$ were not homotopic to a point on $f\left(S^{1} \times I\right)$, then $\lambda$ would be homotopic to a loop on $A$, and this is clearly false. The same reasoning shows that $\mu$ is homotopic to a point on $A$. Then $\mu$ bounds disks $D$ on $f\left(S^{1} \times I\right)$ and $D^{\prime}$ on $A$. We assume $\mu$ chosen an "innermost" loop on $f\left(S^{1} \times I\right)$, i.e., so that $\operatorname{int}(D)$ contains no points of $A$. Now replace $A$ by $\left(A-D^{\prime}\right) \cup D$. See Figure 3 .
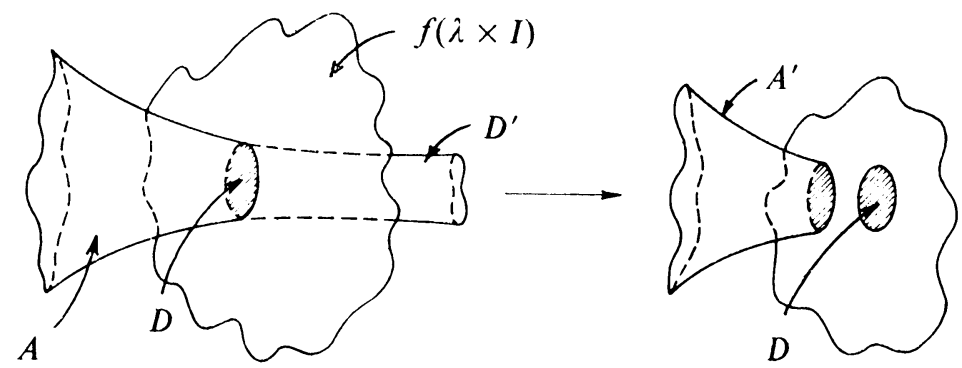

FIGURE 3

By a small motion in a neighborhood of $D$, we can replace $\left(A-D^{\prime}\right) \cup D$ by an annulus $A^{\prime}$, having the same boundary as $A$ and so that

$$
A^{\prime} \cap f\left(S^{1} \times I\right)=\left(A \cap f\left(S^{1} \times I\right)\right)-\mu .
$$

Since $\operatorname{int}(D)$ contains no points of $A$, we have that $A^{\prime}$ is an embedded annulus. Thus by induction we may assume that $A$ is chosen so that $A \cap f\left(S^{1} \times I\right)$ consists entirely of disjoint simple arcs.

A simple arc with its endpoints on the same end of an annulus, cuts off a disk from that annulus. Choose a simple arc $\alpha$ in $A \cap f\left(S^{1} \times I\right)$ such that the disk $D$ in $f\left(S^{1} \times I\right)$ cut off by $\alpha$ contains no points of $A$ in its interior. Let $D^{\prime}$ be the disk on $A$ cut off by $\alpha$, and form $\left(A-D^{\prime}\right) \cup D$. Since $D$ contains no points of $A$ in its interior, this is a regularly embedded annulus in $M \times I$ (see Figure 4).
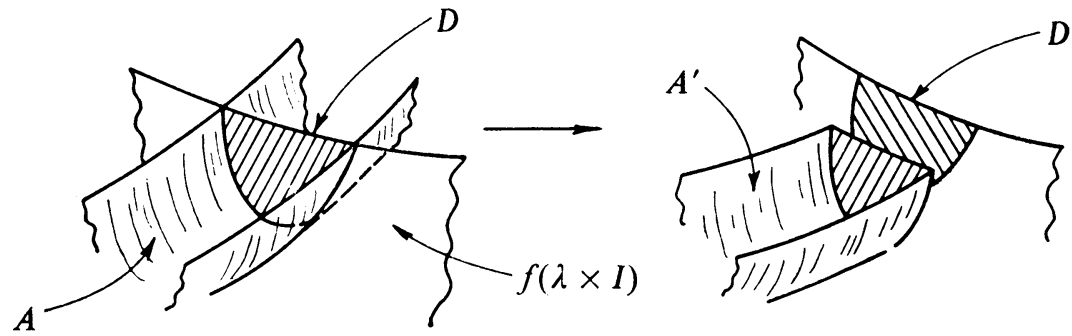

Figure 4 
By a small motion in a neighborhood of $D$, we can displace $\left(A-D^{\prime}\right) \cup D$ to a new position $A^{\prime}$ so that $A^{\prime}$ is regularly embedded in $M \times I$ and

$$
A^{\prime} \cap f\left(S^{1} \times I\right)=A \cap f\left(S^{1} \times I\right)-\alpha .
$$

By induction we see that it is possible to choose $A$ disjoint from $f\left(S^{1} \times I\right)$.

Let $N$ be the closure of the component of $M-\delta$ which contains $\lambda$. Let $h_{1}$ be a homeomorphism of $M \times I$ onto itself which is the identity map on $M \times 0 \cup(\partial M) \times I$, and which carries $A$ onto $\delta \times I$. This exists by Case I, since $\delta$ is a non-null-homotopic separating curve. Let $g=h f$, then $g\left(S^{1} \times I\right)$ is regularly embedded in $N \times I$ since $A$ was disjoint from $f\left(S^{1} \times I\right)$. From now on we shall work with $N$ and $g$. As noted before, $N$ is a torus with a hole in it (see Figure 2).

Choose a simple, nonseparating arc $\beta$ regularly embedded in $N$ with $\beta \cap \lambda=\varnothing$. According to Proposition (2.3) we can find a regularly embedded disk $E$ in $N \times I$ such that

$$
\partial E=\beta \times\{0\} \cup(\partial \beta) \times I \cup(E \cap N \times\{1\})
$$

and $E \cap N \times\{1\}$ is a regularly embedded arc in $N \times\{1\}$. By exactly the same argument as used for the annulus $A$, we can show that $E$ may be chosen disjoint from $g\left(S^{1} \times I\right)$. By Theorem (4.1) we can find a homeomorphism $h_{2}$ of $N \times I$ onto itself which is the identity on $N \times\{0\} \cup(\partial N) \times I$ and so that $h_{2}(E)=\beta \times I$. We extend $h_{2}$ over $M \times I$ to be the identity map on $(M-N) \times I$. Let $l=h_{2} g$.

Let $P$ be the result of cutting $N$ apart along $\beta$; that is, we remove from $N$ the interior of a tubular neighborhood $V$ of $\beta$ in $N$. We assume $V$ chosen so small that $(V \times I) \cap l\left(S_{1} \times I\right)=\varnothing$. Then $l\left(S^{1} \times I\right) \subset P \times I$. Now $P$ is an annulus (see Figure 2), and $\lambda$ is a separating loop in $P$. According to Case $I$ we can find a homeomorphism $h_{3}$ of $P \times I$ onto itself so that $h_{3}$ is the identity on $P \times\{0\} \cup(\partial P) \times I$ and

$$
h_{3} l(\lambda(s), t)=(\lambda(s), t), \quad s \in S^{1}, t \in I .
$$

We extend $h_{3}$ over $(M-P) \times I$ by making it the identity map. Then $h=h_{3} h_{2} h_{1}$ satisfies all requirements of the theorem.

We proceed now to the nonorientable case, and generalize to include both disks and annuli.

(5.2) THEOREM. Let $M$ be a compact 2-manifold (possibly nonorientable), and let $\lambda_{j}, j=1, \cdots, m$, be a finite family of pairwise disjoint, regularly embedded loops in $M$, none of which is null homotopic. Let $\alpha_{k}, k=1, \cdots, n$, be pairwise disjoint arcs, regularly embedded in $M$, and disjoint from the $\lambda_{j}$. Let

$$
f: \bigcup_{j=1}^{m} S^{1} \times I \times\{j\} \cup \bigcup_{k=1}^{m} I \times I \times\{k\} \rightarrow M \times I
$$

be a regular embedding so that 


$$
\begin{aligned}
f(s, o, j) & =\left(\lambda_{j}(s), o\right), & & s \in S^{1}, j=1, \cdots, m, \\
f(t, o, k) & =\left(\alpha_{k}(t), o\right), & & t \in I, \quad k=1, \cdots, n, \\
f(o, t, k) & =\left(\alpha_{k}(o), t\right), & & t \in I, \quad k=1, \cdots, n, \\
f(1, t, k) & =\left(\alpha_{k}(1), t\right), & & t \in I, \quad k=1, \cdots, n,
\end{aligned}
$$

and so that $f \mid \bigcup_{j=1}^{m} S^{1} \times\{1\} \times\{j\} \cup \bigcup_{k=1}^{m} I \times\{1\} \times\{k\}$ is a regular embedding in $M \times\{1\}$. Then there exists a homeomorphism $h$ of $M \times I$ onto itself so that

$$
\begin{aligned}
h f(s, t, j) & =\left(\lambda_{j}(s), t\right), & (s, t) \in S^{1} \times I, \\
h f(s, t, k) & =\left(\alpha_{k}(s), t\right), & (s, t) \in I \times I, \\
h(p, o) & =(p, o), & p \in M, \\
h(p, t) & =(p, t), & p \in \partial M, t \in I .
\end{aligned}
$$

Proof. We proceed by induction on $m+n$. The reduction step for $m+n>1$ is as in (4.1) and (5.1). We need treat only the case $m+n=1$. If $m=0, n=1$ we apply (4.1) while if $n=0, m=1$ and $M$ is orientable we have (5.1). Thus we may assume $M$ is nonorientable, $n=0, m=1$, and we may further assume that $M$ is connected.

Assume that $m=1$ and denote $\lambda_{1}$ by just $\lambda$. Let $V$ be a tubular neighborhood of $\lambda$ in $M$. Define

$$
k: M \times\{0\} \cup(\partial M) \times I \cup V \times I \rightarrow M \times I
$$

by $k \mid M \times\{0\} \cup(\partial M) \times I$ is the inclusion function, $k \mid V \times I$ is a homeomorphism onto a tubular neighborhood of $f\left(S^{1} \times I\right)$ in $M \times I$ such that $k(\lambda(s), t)=f(s, t)$. If we can extend $k$ to a homeomorphism of $M \times I$ onto itself, then $h=k^{-1}$ is the desired homeomorphism.

Let $V^{o}$ be the interior of $V$ in $M$, and let $M_{1}=M-V^{o}$. Let $B=M \times I-k\left(V^{o} \times I\right)$, then $k \mid M_{1} \times\{0\} \cup\left(\partial M_{1}\right) \times I \rightarrow \partial B$ is an embedding, and we shall extend it by applying (3.4). Clearly $B$ is a compact connected manifold, as a submanifold of $M \times I$ it is a Poincaré manifold by (2.1). We must check that $\chi\left(M_{1}\right) \geqq \chi(B)$, and that the map induced by inclusion $\pi_{1}\left(k\left(M_{1}\right) \times\{0\}\right) \rightarrow \pi_{1}(B)$ is an epimorphism. We prove both by proceeding to an orientable double covering. Let $p: \tilde{M} \rightarrow M$ be an orientable double covering of $M$, so $p \times i: \tilde{M} \times I \rightarrow M \times I$ is an orientable double covering of $M \times I$, where $i: I \rightarrow I$ is the identity map. Let $\tilde{M}_{1}=p^{-1}\left(M_{1}\right)$, $\widetilde{B}=(p \times i)^{-1}(B), \tilde{\lambda}=p^{-1}(\lambda)$, and $\tilde{A}=(p \times i)^{-1}\left(f\left(S^{1} \times I\right)\right)$. Then $\tilde{\lambda}, \tilde{A}$ consists of a pair of disjoint loops and a pair of disjoint annuli, or a single loop and a single annulus. In either case the hypotheses of (5.1) are satisfied as long as $M$ is not the projective plane. Leaving this exception aside for the moment there is a homomorphism of $\tilde{M} \times I$ onto itself which is the identity on $\tilde{M} \times\{0\}$ and which carries $\tilde{A}$ onto $\tilde{\lambda} \times I$. Thus the inclusion function $(\tilde{M}-\tilde{\lambda}) \times\{0\} \rightarrow(\tilde{M} \times I)-\tilde{A}$ 
is a homotopy equivalence. In particular $\chi((\tilde{M}-\tilde{\lambda}) \times\{0\})=\chi((\tilde{M} \times I)-\tilde{A})$ and $\pi_{1}((\tilde{M}-\tilde{\lambda}) \times\{0\}) \rightarrow \pi_{1}((\tilde{M} \times I)-\tilde{A})$ is an epimorphism. Since the pair $\left(\tilde{B}, \tilde{M}_{1}\right)$ is a strong deformation retract of the pair $((\tilde{M} \times I)-\tilde{A},(\tilde{M}-\tilde{\lambda}) \times 0)$ we have also $\chi\left(\tilde{M}_{1}\right)=\chi(\widetilde{B})$ and $\pi_{1}\left(\tilde{M}_{1}\right) \rightarrow \pi_{1}(\widetilde{B})$ is an epimorphism. But $2 \chi\left(M_{1}\right)=\chi\left(\tilde{M}_{1}\right)$ and $2 \chi(B)=\chi(\widetilde{B})$ since these are double coverings and have twice as many cells in each dimension. So $\chi\left(M_{1}\right)=\chi(B)$. If $\alpha$ is a path in $B$, with its end points in $M_{1} \times 0$, then $\alpha$ can be lifted to a path in $\widetilde{B}$ with its end points in $\tilde{M}_{1}$. Since $\pi_{1}\left(\tilde{M}_{1}\right) \rightarrow \pi_{1}(\widetilde{B})$ is an epimorphism, we can deform $\tilde{\alpha}$, with end points fixed to a path in $\tilde{M}_{1}$. This deformation covers a deformation of $\alpha$, with end points fixed, to a path in $M_{1}$. Thus $\pi_{1}\left(M_{1}\right) \rightarrow \pi_{1}(B)$ is an epimorphism. For the exceptional case where $M$ is the projective plane, $\tilde{M}$ is a 2 -sphere and $\tilde{\lambda}$ is null-homotopic. Then $\tilde{A}$ separates $\tilde{M} \times I$ into two components, which are easily seen to be cells, each of which covers $B$. Then $\chi\left(M_{1}\right)=\chi(B)=1$, and $\pi_{1}(B)$ is trivial. In any case we can apply theorem (3.4) to the pair $\left(M_{1}, B\right)$ (note that $M_{1}$ is not a projective plane) and this concludes the proof.

6. The unknotting of ares. Our first theorem in this section is also the main result of the section. It gives a condition for the simultaneous unknotting of a family of arcs in $M \times I$. The theorem states that a certain condition on a mapping of the fundamental group of $M \times\{0\}$ less the initial points of the arcs is both necessary and suffcient for the unknotting. There is no reason to prefer $M \times\{0\}$ in the statement of the theorem. If $t, 0 \leqq t \leqq 1$, is any number such that the surface $M \times\{t\}$ intersects each arc in one point, the same condition on the fundamental group of $M \times\{t\}$ less these points is also both necessary and sufficient.

Another remark we might make is that we restrict $M$ to be connected only for convenience in the statement of the theorem. One may allow $M$ to be nonconnected and replace the condition on the fundamental group by a condition on the fundamental group of each component, or by an hypotheseis about deformations of loops.

(6.1) THEOREM. Let $M$ be a compact connected 2-manifold, and let $\alpha_{j}, j=1, \cdots, n$, be a finite number of pairwise disjoint simple arcs regularly embedded in $M \times I$. We suppose $\alpha_{j}(i) \in \operatorname{int}(M \times\{i\})$ for $i=0,1$. If

$$
\pi_{1}\left(M \times\{0\}-\bigcup_{j=1}^{n} \alpha_{j}(0)\right) \rightarrow \pi_{1}\left(M \times I-\bigcup_{j=1}^{n} \alpha_{j}(I)\right)
$$

is onto, then there exists a homeomorphism $h$ of $M \times I$ onto itself such that

$$
\begin{array}{lr}
h(p, 0)=(p, 0), & p \in M, \\
h(p, t)=(p, t), & p \in \partial M, \quad t \in I, \\
h\left(\alpha_{j}(t)\right)=\left(p_{j}, t\right) \text { where } \alpha_{j}(0)=\left(p_{j}, 0\right), & j=1, \cdots, n .
\end{array}
$$


Proof. The arcs $\alpha_{j}$ have arbitrarily small tubular neighborhoods in $M \times I$, in fact by Whitehead [10], we may choose the second regular neighborhood in a sufficiently fine subdivision of $M \times I$. Thus there exist pairwise disjoint disks $D_{j}$ about $p_{j}$ in the interior of $M$ and an embedding $k: \bigcup_{j=1}^{n} D \times I \rightarrow M \times I$ so that

$$
\begin{aligned}
k \mid D_{j} \times 0 & =\text { identity, } \\
k\left(p_{j}, s\right) & =\alpha_{j}(s), \\
k\left(D_{j} \times\{1\}\right) & \subset \operatorname{int}(M \times\{1\}), \\
k\left(D_{j} \times(0,1)\right) & \subset(\operatorname{int} M) \times(0,1) .
\end{aligned}
$$

We further define $k \mid M \times 0 \cup(\partial M) \times I$ to be the identity map. Our only problem now is to extend $k$ over $M \times I$ and $h=k^{-1}$ is as desired.

Let $N=M-\bigcup_{j=1}^{n} \operatorname{int}\left(D_{j}\right)$, and let $B=M \times I-k\left(\bigcup_{j=1}^{n} \operatorname{int}\left(D_{j}\right) \times I\right)$. Then $k \mid N \times 0 \cup(\partial N) \times I$ is a homeomorphism onto a proper subset of $\partial B$. We extend it to all of $N \times I$ by Theorem (3.4). To apply Theorem (3.4) we must show that $\chi(N) \geqq \chi(B)$, and that $\pi_{1}(N \times\{0\}) \rightarrow \pi_{1}(B)$ is onto. (Clearly $B$ is a Poincaré manifold and $N$ is not the projective plane.) If $N_{1}=M \times I-k\left(\bigcup_{j=1}^{n}\right.$ int $\left.D_{j} \times 1\right)$ then $\chi(N)=\chi\left(N_{1}\right)$, and by considering the double of $B$ we see that $2 \chi(B)$ $=\chi(N)+\chi\left(N_{1}\right)$. Thus $\chi(N)=\chi(B)$. On the other hand, the pair $(B, N \times\{0\})$ is a strong deformation retract of the pair $\left((M \times I)-\bigcup_{j=1}^{n} \alpha_{j}(I),(M \times\{0\})-\bigcup_{j=1}^{n} \alpha_{j}(0)\right)$. Thus by hypothesis we have $\pi_{1}(N \times\{0\}) \rightarrow \pi_{1}(B)$ is onto. We may now use (3.4) to extend $k$ over $N \times I$ and hence over $M \times I$. This completes the proof.

As we claimed in the introduction this yields the classical result that a simple closed curve $\lambda$ in $S^{3}$, the 3 -sphere, is unknotted provided the fundamental group of its complement is infinite cyclic. To see this pick a tubular neighborhood of $\lambda$ and remove the interior of the part of the tubular neighborhood around a segment of $\lambda$. That is, we remove the interior of a closed cell from $S^{3}$. We are left with a closed cell, that is $D^{2} \times I$ where $D^{2}$ is a closed disk, and an arc $\alpha$ regularly embedded in $D^{2} \times I$ going from $(p, 0)$ to $(p, 1)$ where $p$ is some interior point of $D$. Then $\lambda$ is unknotted if and only if we can find a homeomorphism of $D^{2} \times I$ onto itself, leaving $\partial\left(D^{2} \times I\right)$ pointwise fixed and carrying $\alpha$ to $\{p\} \times I$. It is enough, however, to find a homeomorphism which leaves $D^{2} \times\{0\} \cup\left(\partial D^{2}\right) \times I$ pointwise fixed and carries $\alpha$ to $\{p\} \times I$. For then one can force it to leave $D \times\{1\}$ pointwise fixed also. Thus we are in a position to apply Theorem (6.1), and we conclude that $\lambda$ is unknotted if and only if the natural homomorphism of the infinite cyclic group $\pi_{1}\left(D^{2} \times\{0\}-(p, 0)\right)$ to $\pi_{1}\left(D^{2} \times I-\alpha\right)$ is onto. That is to say, using Van Kampen's theorem, $\lambda$ is unknotted if and only if the "perpendicular loop" $\mu=\partial D^{2} \times\{0\}$ generates $\pi_{1}\left(S^{3}-\lambda\right)$. Since the homology class of $\mu$ generates $H_{1}\left(S^{3}-\lambda\right)$ in any case, $\lambda$ is unknotted if and only if $\pi_{1}\left(S^{3}-\lambda\right)$ is infinite cyclic.

The situation for links however, is not so simple. If we replace $\lambda$ by a finite number of pairwise disjoint loops, this has the effect of replacing $\alpha$ by a finite 
number of pairwise disjoint arcs. However a homeomorphism of $D^{2} \times I$ onto itself which is the identity on $\left(D^{2} \times\{0\}\right) \cup\left(\left(\partial D^{2}\right) \times I\right)$, and which maps the arcs to product lines, cannot be forced to be the identity on $D^{2} \times\{1\}$. To get an unknotting criterion for links some further hypothesis is necessary.

We now return to something postponed in $\$ 5$, that is, the problem of unknotting an annulus whose base curve is homotopic to a point. We state and prove an unknotting theorem for a single annulus, it is clear from the proof that it extends to a finite number of annuli.

(6.2) THEOREM. Let $M$ be a compact 2-manifold, $\lambda$ a simple, regularly embedded loop in $M$ which is null-homotopic, and let $f: \lambda \times I \rightarrow M \times I$ be a regular embedding such that $f \mid \lambda \times\{0\}$ is the identity, and $f \mid \lambda \times\{1\}$ is a regular embedding in $M \times\{1\}$. Then there exists a homeomorphism $h$ of $M \times I$ onto itself such that

$$
\begin{aligned}
h \mid M \times\{0\} \cup(\partial M) \times I & =\text { identity, } \\
h f(\lambda(s), t) & =(\lambda(s), t) .
\end{aligned}
$$

if and only if every path in $M \times I-f(\lambda \times I)$ with its end points in $M \times\{0\}-(\lambda \times\{0\})$ can be deformed over $M \times I-f(\lambda \times I)$, with end points fixed, to a path in $M \times\{0\}-f(\lambda \times\{0\})$.

Proof. As far as the necessity of the condition goes, it is obvious, and we shall only prove the sufficiency.

The loop $\lambda$ is null-homotopic in $M$, so it is null-homologous in $M$. Thus $M-\lambda$ has two components, whose closures we denote by $N$ and $P$. If neither $N$ nor $P$ were a 2-cell, then the homotopy class of $\lambda$ would be of infinite order in the fundamental group of each of these spaces, since $\lambda$ lies on the boundary of each. Then by Van Kampen's theorem $\lambda$ would not be null-homotopic in $M$. Let us choose notation so that $P$ is a 2 -cell.

Let $A=f(\lambda \times I)$, we claim that $A$ disconnects $M \times I$. For otherwise we could choose a path in $M \times I-A$ joining a point of int $N \times\{0\}$ to a point of int $P \times\{0\}$. By our hypothesis this path could be deformed, with end points fixed, to lie in $M-\lambda \times\{0\}$, an obvious contradiction. Let the closures of the components of $M \times I-A$ be denoted $B$ and $C, B$ containing $N \times\{0\}$. By our hypothesis both $\pi_{1}(N \times\{0\}) \rightarrow \pi_{1}(B)$ and $\pi_{1}(P \times\{0\}) \rightarrow \pi_{1}(C)$ are onto. Further $\chi(M \times I)$ $=\chi(B)+\chi(C)$ and $\chi(M)=\chi(N)+\chi(P)$. Thus either $\chi(N) \geqq \chi(B)$ or $\chi(P) \geqq \chi(C)$. Suppose that $\chi(N) \geqq \chi(B)$. Then by (3.4) $B$ is homeomorphic to $N \times I$ and hence $\chi(N)=\chi(B)$ and $\chi(P)=\chi(C)$. Similarly if $\chi(P) \geqq \chi(C)$.

Consider the mapping $k$ from $M \times\{0\} \cup(\partial M) \times I \cup \lambda \times I$ into $M \times I$ defined by

$$
\begin{aligned}
k \mid M \times\{0\} \cup(\partial M) \times I & =\text { identity } \\
k(\lambda(s), t) & =f(\lambda(s), t) .
\end{aligned}
$$


We have that $N, B, k \mid N \times\{0\} \cup(\partial N) \times I$ and $P, C, k \mid P \times\{0\} \cup(\partial P) \times I$ satisfy all hypotheses of (3.4). Thus we can extend $k$ to a homeomorphism of $M \times I$ onto itself, and $h=k^{-1}$ is as desired. The proof is complete.

In the above proof we see that $C$ is homeomorphic to $P \times I$, a 3-cell. Thus there is a 3-cell $C$ in $M \times I$ whose boundary is $A \cup(P \times\{0\}) \cup(C \cap M \times\{1\})$. Now the existence of this cell does not depend on the deformation hypothesis of (6.2), but only on the fact that $\lambda$ is null-homotopic. For consider the disk $A \cup(P \times\{0\})$. It is not hard to see that this disk separates $M \times I$, let the closures of the components of its complement be $B$ and $C, C$ containing $P \times\{0\}$. We assert that $C$ is a cell. First $\pi_{1}(M) \cong \pi_{1}(M \times I) \cong \pi_{1}(B \cup(P \times\{0\})) * \pi_{1}(C)$, and the map $\pi_{1}(B \cup(P \times\{0\})) \rightarrow \pi_{1}(M \times I)$ is onto since even $\pi_{1}(M \times\{0\}) \rightarrow \pi_{1}(M \times I)$ is onto. Thus $\pi_{1}(C)=1$, it follows that $C \cap M \times\{1\}$ has no nontrivial loops, i.e. that it is a disk. Thus $\partial C$ is a 2 -sphere and we conclude that $C$ is a 3-cell since $M \times I$ is a Poincaré manifold. Thus we can extend $f$ to a homeomorphism $h: P \times I \rightarrow M \times I$ so that $h \mid P \times\{0\}=$ identity and $h(P \times\{1\}) \subset M \times\{1\}$. For any such extension, and any point $p$ in $\operatorname{int}(P)$ we refer to $h(\{p\} \times I)$ as a core of $f(\lambda \times I)$. Then we have

(6.3) THEOREM. Let $M, \lambda$ and $f$ be as in (6.2), but do not assume the deformation hypothesis. Then the conclusion still holds provided $f(\lambda \times I)$ has an unknotted core; that is a core $h(\{p\} \times I)$ which satisfies the hypotheses of (6.1).

Proof. We need only note that in the proof of (6.1) we can choose $h(P \times I)$ as the tubular neighborhood of $h(\{p\} \times I)$.

This last result may be paraphrased by saying that a null-homotopic annulus in $M \times I$ is unknotted if and only if it bounds an unknotted piece of rope.

We may expand or contract $C$ slightly, by using a tubular neighborhood of $f(\lambda \times I)$ in $M \times I$, without changing whether or not $f(\lambda \times I)$ is unknotted. Thus we may use $f(\{p\} \times I)$ as a representative core for $f(\lambda \times I)$, where $p$ is any point on $\lambda$. Putting this together with (6.1) we get the last theorem of this section.

(6.4) TheOrem. Let $M$ be a compact connected 2-manifold, $\lambda$ a null-homotopic simple loop on $M$. Let $f: \lambda \times I \rightarrow M \times I$ be a regular embedding so that $f(\lambda \times\{1\})$ is regularly embedded in $M \times\{1\}$, and $f(\lambda(s), 0)=(\lambda(s), 0)$. Then there exists a homeomorphism $h$ of $M \times I$ onto itself so that

$$
\begin{aligned}
h \mid M \times\{0\} \cup(\partial M) \times I & =\text { identity, } \\
h f(\lambda(s), t) & =(\lambda(s), t),
\end{aligned}
$$

if and only if for some point $p$ in $\lambda$, the homomorphism

$$
\pi_{1}(M \times\{0\}-(p, 0)) \rightarrow \pi_{1}(M \times I-f(\{p\} \times I))
$$

induced by the inclusion map is onto. 
7. The unknotting of $M$. In this section we shall consider the unknotting of $M$ in $M \times I$. The case where $M$ is a 2 -sphere is essentially Alexander's theorem. The case where $M$ is a torus was first solved by C. H. Edwards, and more recently he extended this to all compact, nonbounded manifolds [2]. Our proof makes no distinction between the bounded and the nonbounded case, so it provides a new proof of Edwards' theorem. According to our Theorem (3.4) the proof is, with the exception of $M$ a projective plane, almost entirely algebraic. The next theorem is the algebraic heart of the unknotting theorem.

(7.1) THEOREM. Let $M, N$ be compact, connected, 2-manifolds with homeomorphic boundaries, and let $f: N \rightarrow M \times I$ be a regular embedding such that $f(N)$ separates $M \times\{0\}$ from $M \times\{1\}$. Denote the closures of the components of $M \times I-f(N)$ by $C_{0}$ and $C_{1}, C_{j} \supset M \times\{j\}$. Then we have

(i) $\chi(N) \leqq \chi(M)$.

(ii) If $N=M$ then $\pi_{1}(f(N)) \rightarrow \pi_{1}\left(C_{j}\right)$ is a monomorphism for $j=0$ and for $j=1$.

(iii) If $\chi(N)<\chi(M)$ then $\pi_{1}(f(N)) \rightarrow \pi_{1}\left(C_{j}\right)$ cannot be a monomorphism for both $j=0$ and $j=1$.

(iv) If $\pi_{1}(f(N)) \rightarrow \pi_{1}\left(C_{j}\right)$ is a monomorphism for both $j=0$ and for $j=1$ then $\pi_{1}(f(N)) \rightarrow \pi_{1}\left(C_{J}\right)$ is an isomorphism in both cases.

Proof. We start by proving (iv). If both maps are monomorphisms then by Van Kampen $\pi_{1}(M \times I) \cong \pi_{1}\left(C_{0}\right) *_{\pi_{1}(f(N))} \pi_{1}\left(C_{1}\right)$. Thus the maps $\pi_{1}\left(C_{j}\right) \rightarrow \pi_{1}(M \times I)$ are monomorphisms. They are also epimorphisms since the composition $\pi_{1}(M \times\{j\}) \rightarrow \pi_{1}\left(C_{j}\right) \rightarrow \pi_{1}(M \times I)$ is an epimorphism. Using the theorem quoted from Hall [4, p. 314], which appears just before Proposition (2.5), it is immediate that $\pi_{1}(f(N)) \rightarrow \pi_{1}\left(C_{J}\right)$ is an epimorphism for $j=0,1$. Notice that (iii) follows now since we may further conclude that

$$
\pi_{1}(f(N)) \cong \pi_{1}\left(C_{0}\right) \cong \pi_{1}(M \times I) \cong \pi_{1}(M)
$$

and this cannot happen if $\chi(N)<\chi(M)$.

We prove (i) and (ii) simultaneously, by supposing that $\chi(N) \geqq \chi(M)$ and showing $\pi_{1}(f(N)) \rightarrow \pi_{1}\left(C_{j}\right)$ is a monomorphism for both $j=0$ and $j=1$. It then follows as above that $\chi(N)>\chi(M)$ is impossible, so $\chi(N) \leqq \chi(M)$. The proof is in two cases according as $M$ is orientable or not.

Case I. $\quad M$ is orientable, and suppose that $\pi_{1}(f(N)) \rightarrow \pi_{1}\left(C_{0}\right)$ is not a monomorphism. Then according to the loop theorem [9], there is a regularly embedded disk $D \subset C_{0}$ with its boundary a regularly embedded, non-null-homotopic loop in $f(N)$. We thicken $D$ and remove its interior; that is find an embedding $g: D \times[-1,1] \rightarrow C_{0}$ so that

$$
\begin{aligned}
g((\text { int } D) \times[-1,1]) & \subset \operatorname{int} C_{0}, \\
g((\partial D) \times[-1,1]) & \subset \operatorname{int} f(N), \\
g(d, 0) & =d \quad \text { all } d \in D,
\end{aligned}
$$


Now let

$$
P=(f(N)-g((\partial D) \times[-1,1])) \cup g(D \times\{-1\}) \cup g(D \times\{1\}) .
$$

Then $\chi(P)=\chi(f(N))+2>\chi(f(N))$, and we claim that $P$ also separates $M \times I$. Suppose to the contrary that there is a path $\alpha$ from $M \times\{0\}$ to $M \times\{1\}$ not meeting $P$. On the one hand, the intersection number of $\alpha$ with $f(N)$ must be odd since $\alpha$ starts in one component of the complement of $f(N)$ and ends in the other. On the other hand, $\alpha$ meets $f(N)$ only in points of $g((\partial D) \times[-1,1])$ since $\alpha \cap P=\varnothing$. But $\alpha$ starts and ends outside the cell $g(D \times[-1,1])$ so $\alpha$ has even intersection number with its boundary, and meets its boundary only in points of $g((\partial D) \times[-1,1])$. This contradiction shows that $P$ still separates $M \times\{0\}$ from $M \times\{1\}$.

Now $P$ may be connected or not, we claim that one of the components of $P$ separates $M \times\{0\}$ from $M \times\{1\}$. Suppose then that $P$ has two components $P_{1}$ and $P_{2}$, and that $P_{2}$ does not separate $M \times\{0\}$ from $M \times\{1\}$. We consider first the case where $\partial M=\varnothing$, and embed $M \times I$ in $R^{3}$ (possible since $M$ is orientable). Then $P_{1}$ and $P_{2}$ separate $R^{3}$ since they are homology cycles, and hence boundaries. Let $E$ be the component of $R^{3}-P_{2}$ which contains both $M \times\{0\}$ and $M \times\{1\}$. Since $P$ separates $M \times\{0\}$ from $M \times\{1\}, P_{1}$ lies in $E$ and separates $M \times\{0\}$ from $M \times\{1\}$ in $E$. But then $P_{1}$ separates $M \times\{0\}$ from $M \times\{1\}$ in $R^{3}$ and hence in $M \times I$.

Now suppose $\partial M \neq \varnothing$. Since $M, N$ and hence $P$ have homeomorphic boundaries, for each boundary component $C_{i}$ of $M$ there is a boundary component $C_{i}^{\prime}$ of $P$, so that $C_{i}^{\prime}$ separates $C_{i} \times\{0\}$ from $C_{i} \times\{1\}$ in $C_{i} \times I$. We "close" $M$ by attaching a disk $D_{i}$ to each boundary component $C_{i}$. We also close $P$ by attaching to each $C_{i}^{\prime}$ a disk $D_{i}^{\prime}$ regularly embedded in $D_{i} \times I$ and separating $D_{i} \times\{0\}$ from $D_{i} \times\{1\}$. Using $M^{*}$ and $P^{*}$ to denote the "closures" of $M$ and $P$, we have that $P^{*}$ separates $M^{*} \times\{0\}$ from $M^{*} \times\{1\}$ in $M^{*} \times I$. By the nonbounded case it follows easily that $P_{1}^{*}$ separates $M^{*} \times\{0\}$ from $M^{*} \times\{1\}$. If $\alpha$ is an arc in $M \times I$ joining $M \times\{0\}$ to $M \times\{1\}$ then $\alpha$ must meet $P_{1}$. But $P_{1}^{*} \cap M \times I=P_{1}$ so $\alpha$ meets $P_{1}$. Thus $P_{1}$ separates $M \times\{0\}$ from $M \times\{1\}$ in $M \times I$. In particular $\partial P=\partial P_{1}$.

We claim next that $\chi\left(P_{1}\right)>\chi(N)$. For $\partial P_{2}=\varnothing$ and $P_{2}$ is not a 2 -sphere since $\partial D$ is not null-homotopic in $f(N)$. Thus $\chi\left(P_{2}\right) \leqq 0$ and $\chi(N)<\chi(P)=\chi\left(P_{1}\right)$ $+\chi\left(P_{2}\right) \leqq \chi\left(P_{1}\right)$. Thus in any case some component $P_{1}$ of $P$, having greater characteristic than that of $N$, separates $M \times\{0\}$ from $M \times\{1\}$. Since the characteristic has actually gone up, we may argue by induction on $\chi(N)$, that the fundemental group of $P_{1}$ maps monomorphically into the fundamental groups of each of its complements. Applying (iv) now we conclude that $\pi_{1}\left(P_{1}\right)$ is isomorphic to $\pi_{1}(M)$ so $P_{1}$ is homeomorphic to $M$. But $\chi(M) \leqq \chi(N)<\chi\left(P_{1}\right)$ which is a contradiction. This contradiction finishes the orientable case.

Case II. $M$ is not orientable. In this case we use the same type of argument that we have used several times already. That is, we pass to an orientable double 
covering of $M$ and of $M \times I$. The points over $f(N)$ form an orientable double cover of $f(N)$ and also separate. Then the maps of the fundamental groups in the covering are monomorphisms by Case I, so those of $\pi_{1}(f(N))$ into $\pi_{1}\left(C_{j}\right)$ are also monomorphisms. This concludes the proof.

(7.2) THEOREM. Let $M$ be a compact 2-manifold, $f: M \rightarrow M \times I$ a regular embedding such that $f(M)$ separates $M \times\{0\}$ from $M \times\{1\}$. Then there exists a homeomorphism $h$ of $M \times I$ onto itself such that $h f(m)=(m, 1 / 2)$ for all $m \in M$.

Proof. We note first that $M$ and $M \times I$ have the same number of components, so $f$ maps components of $M$ one-to-one to components of $M \times I$. If $M_{i}$ and $M_{j}$ are components of $M$, and $f\left(M_{i}\right) \subset M_{j} \times I$, then by (7.1) part (i) we have that $\chi\left(M_{i}\right) \leqq \chi\left(M_{j}\right)$. Since this is true for every $M_{i}$ we must have $\chi\left(M_{i}\right)=\chi\left(M_{j}\right)$, that is, $M_{i}$ and $M_{j}$ are homeomorphic. From this we may, and shall, assume that $M$ is connected.

Let the closures of the two components of $M \times I-f(M)$ be $C_{0}$ and $C_{1}, C_{j} \subset M \times\{i\}$. According to (7.1) parts (ii) and (iv) the maps $\pi_{1}(f(M)) \rightarrow \pi_{1}\left(C_{j}\right)$ are onto for $j=0$, 1. Moreover $\chi(M)=\chi\left(C_{0}\right)+\chi\left(C_{1}\right)-\chi(f(M))$, so either $\chi(f(M)) \geqq \chi\left(C_{0}\right)$ or $\chi(f(M)) \geqq \chi\left(C_{1}\right)$. Suppose $\chi(f(M)) \geqq \chi\left(C_{0}\right)$. Define a map $k: M \times\{1 / 2\} \rightarrow \partial C_{0}$ by $k(m, 1 / 2)=f(m)$. Extend $k$ to a homeomorphism of $\partial(M) \times[0,1 / 2]$ onto $C_{0} \cap((\partial M) \times I)$ which can be done since each component is an annulus.

Case I. Suppose $M$ is not the projective plane. Since $C_{0} \subset M \times I$ it is a Poincaré manifold so we may apply (3.4) to extend $k$ to a homeomorphism of $M \times[0,1 / 2]$ onto $C_{0}$.

In particular this shows that $\chi(f(M))=\chi\left(C_{0}\right)$ so $\chi(f(M))=\chi\left(C_{1}\right)$ also. We may apply (3.4) again to extend $k$ to a homeomorphism of $M \times[1 / 2,1]$ onto $C_{1}$ so as to carry $M \times\{1\}$ onto $M \times\{1\}$. The inverse of this total extension is the homeomorphism $h$ as desired.

Case II. $M$ is the projective plane. Choose a simple loop $\lambda$ on $M$ which generates the fundamental group and such that $\lambda \times I$ is in general position with respect to $f(M)$. Then $(\lambda \times I) \cap f(M)$ is a finite number of pairwise disjoint simple loops. Since $(\lambda \times I) \cap f(M)$ disconnects $\lambda \times\{0\}$ from $\lambda \times\{1\}$, by (2.2) at least one of these loops, say $\lambda_{1}$, separates $\lambda \times\{0\}$ from $\lambda \times\{1\}$. Then $\lambda_{1}$ is not null-homotopic in $M \times I$, and hence $\lambda_{1}$ is not null-homotopic on $f(M)$. On the other hand, two non-null-homotopic loops in a projective plane must intersect. Thus if $\lambda_{i}$ is any other loop in $f(M) \cap(\lambda \times I)$, we must have that $\lambda_{i}$ is null-homotopic on both $f(M)$ and on $\lambda \times I$. Thus each $\lambda_{i} \neq \lambda_{1}$ bounds disks $D$ and $D^{\prime}$ on $f(M)$ and $\lambda \times I$ respectively. Choose $\lambda_{i}$ so that the disk $D$ it bounds on $f(M)$ contains no other points of $\lambda \times I$. Replace $\lambda \times I$ by $\left(\lambda \times I-D^{\prime}\right) \cup D$, and by a small motion in a neighborhood of $D$ we get a new annulus $A^{\prime}$ such that $A^{\prime} \cap f(M)=((\lambda \times I) \cap f(M))-\lambda_{i}$ and $A^{\prime} \cap M \times\{j\}=\lambda \times\{j\}$ for $j=0,1$. 
Continuing in this way we arrive at an annulus $A$ in $M \times I$ such that $A \cap f(M)=\lambda_{1}$ and $A \cap M \times\{j\}=\lambda \times\{j\}$ for $j=0,1$. According to (5.2) there is a homeomorphism $g$ of $M \times I$ onto itself such that $g(A)=\lambda \times I$.

To prove that $k$ can be extended it is sufficient to prove that $C_{0}$ and $C_{1}$ are homeomorphic to $M \times I$, equivalently that $g\left(C_{0}\right)$ and $g\left(C_{1}\right)$ are homeomorphic to $M \times I$. But if we cut $M \times I$ apart along $\lambda \times I$ we get $N \times I$, where $N$ is the disk that results from cutting $M$ apart along $\lambda$. Furthermore this cuts $g f(M)$ apart along $g\left(\lambda_{1}\right)$, the result is a disk regularly embedded in $N \times I$ and satisfying the hypotheses of this theorem. According to Case I, the result of cutting $g\left(C_{0}\right)$ apart along $\lambda \times I \cap g\left(C_{0}\right)$ is a cell. Thus if we extend $g k$ over $\lambda \times[0,1 / 2]$ to a homeomorphism onto $\lambda \times I \cap g\left(C_{0}\right)$, we can further extended it to a homeomorphism of $M \times[0,1 / 2]$ onto $g\left(C_{0}\right)$. Similarly $g\left(C_{1}\right)$ is homeomorphic to $M \times I$ and the proof is complete.

\section{REFERENCES}

1. E. M. Brown and R. H. Crowell, Deformation rectractions of 3-manifolds into their boundaries, Ann. of Math. 82 (1965), 445-458.

2. C. H. Edwards, Concentricity in 3-manifolds, Trans. Amer. Math. Soc. 113 (1964), 406-423.

3. V. K. A. M. Gugenheim, Piecewise linear isotopy and embedding of elements and spheres. I, Proc. London Math. Soc. (3) 3 (1953), 29-53.

4. M. Hall, The theory of groups, Macmillan, New York, 1959.

5. P. J. Hilton and S. Wylie, Homology theory, Cambridge Univ. Press, New York, 1960.

6. C. D. Papakyriakopoulos, Dehn's lemma and the asphericity of knots, Ann. of Math. 66(1957), 1-26.

7. A. Shapiro and J. H. C. Whitehead, A proof and extension of Dehn's lemma, Bull. Amer. Math. Soc. 64(1958), 174-178.

8. J. Stallings, On fibering certain 3-manifolds, Topology of 3-manifolds and related topics, Prentice-Hall, Englewood Cliffs, N. J., 1961.

9. _- On the loop theorem, Ann. of Math. 72 (1960), 12-19.

10. J. H. C. Whitehead, Simplicial spaces, nuclei, and m-groups, Proc. London Math. Soc. (2) 45 (1939), 243-327.

Dartmouth College, HANOVER, New HAM PSHIRE 